\title{
Physics of superfluid helium-4 vortex tangles in normal-fluid strain fields
}

\author{
Demosthenes Kivotides $\oplus^{1}$ and Anthony Leonard ${ }^{2}$ \\ ${ }^{1}$ Chemical Engineering, Strathclyde University, Glasgow G1 1XL, United Kingdom \\ ${ }^{2}$ California Institute of Technology, Pasadena, California 91125, USA
}

(Received 4 October 2020; accepted 9 April 2021; published 21 April 2021)

\begin{abstract}
By employing dimensional analysis, we scale the equations of the mesoscopic model of finite temperature superfluid hydrodynamics. Based on this scaling, we set up three problems that depict the effects of kinematic, normal-fluid strain fields on superfluid vortex loops, and characterize small-scale processes in fully developed turbulence. We also develop a formula for the computation of energy spectra corresponding to superfluid vortex tangles in unbounded domains. Employing this formula, we compute energy spectra of superfluid vortex patterns induced by uniaxial, equibiaxial, and simple-shear normal-fluid flows. By comparing the steady-state superfluid spectra and vortex structures, we conclude that normal-flow strain fields do not play an important role in explaining the phenomenology of fully developed superfluid turbulence. This is in sharp contrast with the role of vortical normal-flow fields in offering plausible, structural explanations of superfluid vortex patterns and spectra entailed in numerical turbulent solutions of the mesoscopic model.
\end{abstract}

DOI: 10.1103/PhysRevFluids.6.044702

\section{INTRODUCTION}

The advent of quantum theory posed the problem of quantizing fluid dynamics. The most obvious choice, i.e., quantizing the Navier-Stokes fluid, leads to unsurpassable mathematical difficulties with uncontrollable singularities [1]. This could be because Navier-Stokes is a statistical, dissipative field theory and, traditionally, quantum mechanics was successful operating on conservative classical field theories. Indeed, things become better with the quantization of (nonlinear) Schrödinger (NLS) type fluids, that can explain superfluidity via spontaneous breaking of the particle number symmetry which induces a ground state (superfluid) whose topological defects (superfluid vortices) interact with the fluctuations of the quantum field (normal fluid) [2-7]. The superfluid has a hydrodynamic description at all scales, while the fluctuations have a much richer phenomenology: they can be studied at the level of quantum field theory [8], or in the realm of statistical physics, with quantum kinetic theory $[9,10]$ and hydrodynamics, employing, in the latter case, either the mesoscopic model (MM) [11,12] or its statistical mechanics [7,13-19], which we refer to here as effective continuum model (ECM). A key, and historically important, example of ECM is the celebrated Hall-VinenBekarevich-Khalatnikov equations. An excellent discussion of this crucial physics is available in book form [20]. The first two approaches provide very detailed physics information that is not required in many technological and astrophysical applications of superfluids. On the other hand, ECM equations need to be tailored to the particular flows in mind, and are not expected to have a general validity. In this sense, the MM, having been based on simpler principles of fluid physics, and by not having the complexity of combined NLS-kinetic theory formulations, strikes a balance between general validity and computational complexity. Indeed, as discussed in [11,12] there is an analogy between superfluids and complex fluids that ensures the consistency and completeness of the MM. The analogy is not exact since, while the mass of a polymer system is localized at polymer 
positions, the "mass" attributed to a vortex is a quantity distributed over fluid volumes. On the other hand, the relation of ECM with MM is similar to the relation of Reynolds stress models (RSMs) with direct numerical simulations (DNS) in classical turbulence theory. Similar to what happens there, the implications of MM for ECM ought to be a key research topic, which, at present, and not withstanding its technical difficulty, appears to be highly underdeveloped [14,21]. It is important to realize that the defects modeled in the MM are in one-to-one correspondence with those of the NLS equation, since they are discrete, while those modeled in the ECM approaches correspond to coarse-grained superfluid vorticity, i.e., to continuum superfluid vorticity distributions. Because of these, quantities such as vortex line density or intervortex spacing are unambiguously defined within the MM framework, but have no meaning within the ECM one. The above are reflected upon the different sets of mutual friction coefficients employed in the two approaches, since in the ECM approach the coefficients depend upon the flow encountered by the superfluid vortices, while in the MM they are flow independent material properties. This is similar to the difference between the coefficients in the DNS and RSM approaches in classical turbulence. The former employ the material viscosity, while the latter, as, for example, in the case of eddy viscosity models, employ much larger, flow dependent coefficients. This disparity in the magnitude of the coefficients leads to very different flow physics, since the much higher eddy viscosity values damp the instabilities that lead to turbulence, hence RSM equations can only model smooth flow fields evolving over the scale of the turbulent large eddies, and are unable to resolve velocity or pressure fluctuations.

At the mesoscopic level, superfluid vortices lose their small-scale compressibility, and can schematically be described as superfluid vortices = incompressible Euler vortices + reconnections (where the latter were introduced in computational superfluids research in the pioneering contributions of Schwarz [7,22,23]). Notably, the superfluid (quantum vacuum or ground state) is not identical to the condensate, since its energy also includes the zero-point energy of the quasiparticles that comprise the normal fluid $[20,24]$. It is because of this convention that the normal fluid is exclusively associated with thermal effects in the system, since the quasiparticle energy levels (above their zero-point energy) depend on the fluid temperature. The normal fluid obeys a forced Navier-Stokes equation with delta-function forcing due to its interactions with the superfluid vortices (mutual friction). Due to its similarity with complex fluids models, the MM is capable of resolving all hydrodynamic scales in the normal -fluid. This becomes particularly important in light of recent MM results showing that the most interesting interactions between the topological defects of the ground state and the fluctuations occur at the large wave-number end of the normal-fluid turbulence spectrum [11,25]. A key MM achievement is the indication of the important role of interactions between vortices in the two fluids in the generation and structure of superfluid turbulence, and in dissipation processes and enstrophy damping of normal-fluid turbulence [11,12,21,26]. Although a focus on vortex structure is justified by the latter's dominant role in homogeneous, isotropic turbulence phenomenology and energetics, the rotation rate tensor is only part of the object measuring the "strength" of flow effects, i.e., the velocity gradient tensor. The latter involves also the strain rate tensor that, in classical turbulence, characterizes turbulence energy dissipation rate and turbulent material-line and polymer stretching [27,28]. Therefore, it is important to consider the response of superfluid topological defects to generic strain rate fields, which are ubiquitous in turbulent flows, and could help us understand superfluid vortex structures, energy spectra [11], or vortex length dynamics [25] in finite temperature flows. Indeed, a turbulent flow is not so much a special type of flow, as it is an envelope of all possible flow types. One can identify, for example, stagnation points (that correspond to the straining fields studied here) within a fluctuating Navier-Stokes flow field. Depending on their persistence properties, such microflows can have significant dynamical effects. For example, a biextensional flow can flatten vorticity blobs into vortex sheets, hence, providing a $k^{-2}$ spectral signature to the flow, and uniaxial flow fields can be associated with vortex stretching, an important mechanism of energy transfer between flow scales. Our viewpoint here is slightly different, since, unlike the classical turbulence case, we consider the effect of strong normal-fluid strain on superfluid, rather than normal-fluid, vorticity. This is similar with analogous developments in other turbulence physics fields, such as turbulence in polymeric fluids or suspensions where the 
interaction between flow strain and polymers or particles is very important. This article presents the results of such an analysis for simple-shear, uniaxial, and biaxial extensional flows. In this first step, and in order to isolate small timescale physics, we do not include the reaction of the defects on the fluctuations. Such a reaction will be important at mesoscopic to large evolution timescales, and qualifies for further investigation.

\section{MATHEMATICAL MODEL OF FINITE TEMPERATURE SUPERFLUID FLOWS AND ITS SCALING}

We follow the latest version of MM vortex dynamics [12], but with kinematically prescribed normal fluid. Accordingly, the superfluid vortices obey the equation

$$
\dot{\mathbf{X}}_{v}=-\mathbf{X}_{v}^{\prime} \times\left(\mathbf{X}_{v}^{\prime} \times \mathbf{V}_{s}\right)+\alpha \mathbf{X}_{v}^{\prime} \times\left[\mathbf{X}_{v}^{\prime} \times\left(\mathbf{V}_{n}-\mathbf{V}_{s}\right)\right]+\beta \mathbf{X}_{v}^{\prime} \times\left(\mathbf{V}_{n}-\mathbf{V}_{s}\right)
$$

where $\mathbf{X}_{v}(t)$ is a generic position on a superfluid vortex, $\mathbf{V}_{s}$ is the the Biot-Savart superfluid velocity, $\mathbf{X}_{v}^{\prime}$ is the unit tangent vector at a vortex line position, $\mathbf{V}_{n}$ is the normal-fluid velocity on a vortex, $\alpha=-\left[b(1+b)+a^{2}\right] /\left[(1+b)^{2}+a^{2}\right]$, and $\beta=a /\left[(1+b)^{2}+a^{2}\right]$, where $a=D_{\perp} / \rho_{s} \kappa$ and $b=\rho_{n} / \rho_{s}$, and $D_{\perp}$ is a coefficient of the drag force on superfluid vortices that are normal to the difference of vortex and normal-fluid velocities [12]. In our calculations, the values of the dimensionless coefficients are $\alpha=-0.094$ and $\beta=0.211$.

It is important, as a complement to the discussion in [12], to comment here on the physics of the employed vortex dynamics. According to the above mathematical model, three forces act on superfluid vortices: vacuum lift (VL; Magnus) force, viscous friction (VF; Hall-Vinen) force, and quasiparticle lift (QL; Iordanskii) force. All of these forces have been experimentally measured, and are extensively discussed in excellent monographs [20,29]. We have neglected the inertial and elastic-tension forces [11]. We add some important comments on the relevance of the QL force in incompressible quantum fluid turbulence. This force originates in the scattering of quasiparticles by defects in the vacuum. It is anticipated by the fact that quasiparticles are excitations of the vacuum, so they can interact with defects in the latter. Although quasiparticles are quantum objects, previous derivations of QL force employed classical physics. In particular [29], the force was derived in roton scattering processes, where rotons were modeled as classical particles (geometrical optics approximation in wave mechanics), and in phonon scattering processes, where phonons were modeled as sound (linear) waves. Hence, in both cases, the analyses employed the classical objects whose quanta are the corresponding quasiparticles. Indeed, high frequency rotons are more particlelike, while low frequency phonons are more wavelike, but the actual situation involves interactions best described by quantum field theory (QFT) [30]. A remarkable feature of the QFT approach [30] is the derivation, within the same analysis framework, of both VF and QL forces. Both roton and phonon quasiclassical computations give similar results, but the two forces ought not to be summed [29], since they are in fact the same force which encodes the analog of Aharonov-Bohm (AB) effects in superfluids $[20,29]$. The superfluids $A B$ effect is more analogous to the gravimagnetic $A B$ effect rather than the original electromagnetic one. This is because a superfluid vortex and the quasiparticle flow around it, correspond directly to a gravimagnetic flux tube and the fluid flow around it [20,31]. This is a key fact, since, in the phonon case, previous analyses employing sound waves require a compressible normal fluid, and indicate a classical character for the QL force. However, this force can be relevant to the incompressible flows here, because it is a quantum mechanical (AB effect) force, and the waves scattered by the vacuum vortices are quantum probability waves [32]. Moreover, the connection with the AB effect ensures the topological character of QL force [32], hence also its independence from particular approaches employed in its derivation (which explains the similar results obtained for phonons and rotons). The topological nature of the force can be easily understood: since the superfluid is vorticity free, according to Stokes' theorem, the only possibility for it to develop recirculating flow around linear defects and accompanying lift forces, is when its flow domain is not simply connected. 


\section{A. Generic strain dominated flow fields}

Since a uniform flow is dynamically irrelevant (any uniform flow field could be removed via a Galilean transformation), the velocity gradient tensor $G_{i j} \equiv \partial u_{i} / \partial x_{j}$ measures, in a sense, the "strength" of flow effects. Here, $\mathbf{u}(\mathbf{x}, t)$ is the fluid flow velocity, and latin indices denote the directions of Euclidean space. $G_{i j}$ can be decomposed into the sum of a symmetric and an anisymmetric tensor: $G_{i j}=e_{i j}+\omega_{i j}$, where $e_{i j}$ is the strain rate tensor, and $\omega_{i j}$ is the rotation rate tensor, although other names are also in use. $e_{i j} \equiv\left(\partial u_{i} / \partial x_{j}+\partial u_{j} / \partial x_{i}\right) / 2$ and $\omega_{i j} \equiv\left(\partial u_{i} / \partial x_{j}-\partial u_{j} / \partial x_{i}\right) / 2$. Since $\omega_{i j}$ is antisymmetric, it is equivalent to the known pseudovector of flow vorticity $\omega(\mathbf{x}, t)$. The tensor $e_{i j}$, which can be written as $e_{i j}=t_{i j}+d_{i j}$, where $t_{i j}=\delta_{i j} e_{l l} / 3$ is the diagonal, volume rate of expansion tensor and $d_{i j}=e_{i j}-\delta_{i j} e_{l l} / 3$ is the rate of shear strain tensor or deviator. In our case, the normal fluid is incompressible, so there is no difference between the deviator and the rate of strain tensor.

There are three simple flow fields that exemplify key strain physics: uniaxial flow, where a material line is stretched along one dimension and compressed along the other two, equibiaxial flow with one compressing and two stretching flow directions, and simple shear, which is a more compound flow field exemplifying both strain (nonzero deviator) and rotation (nonzero rotation tensor). By definition, if $\epsilon_{i j}$ is the strain tensor, we can write $G_{i j}=\dot{\epsilon}_{i j}$, where the dot over the symbol denotes differentiation with respect to time. Then, if $\dot{\epsilon}$ is a constant strain rate, we have for the case of simple shear

$$
G_{i j}=\left(\begin{array}{ccc}
0 & \dot{\epsilon} & 0 \\
0 & 0 & 0 \\
0 & 0 & 0
\end{array}\right)
$$

and for the two extensional flow cases

$$
G_{i j}=\left(\begin{array}{ccc}
\dot{\epsilon} & 0 & 0 \\
0 & m \dot{\epsilon} & 0 \\
0 & 0 & -(1+m) \dot{\epsilon}
\end{array}\right),
$$

where $m=-0.5$ for uniaxial extensional flow, and $m=1$ for equibiaxial extensional flow. In the calculations, it is $\dot{\epsilon}=10 \mathrm{~s}^{-1}$. The velocity field can easily be computed as $u_{i}(x)=G_{i j} x_{j}$, where $x_{j}$ is the $j$ coordinate of position $x$ in the flow. To test the effects of different initial conditions, we have performed two sets of calculations. The first is called R1 and is the basis of our presentation, hence results presented without additional specification are assumed to be R1 results. The second, called $\mathrm{R} 2$, is mentioned for comparison purposes. In R1, the initial conditions consist of 20 randomly distributed superfluid vortex rings, of typical diameter equal to $D=0.04 \mathrm{~cm}$. In R2, the initial conditions consist of 40 randomly distributed superfluid vortex rings, of typical diameter equal to $D=0.02 \mathrm{~cm}$. The particulars of R2 were chosen so that we have the same initial vortex length in both cases. The rings are placed around the origin of the coordinate system that we use in the definition of the kinematic strain-flow fields. Hence, the origin is a stagnation point in all flows. Employing $D$ and the quantum of circulation $\kappa=9.97 \times 10^{-4} \mathrm{~cm}^{2} \mathrm{~s}^{-1}$, we compute the characteristic superfluid inverse timescale $\tau=0.1557 \mathrm{~s}^{-1}$. This gives a ratio $\chi=\dot{\epsilon} / \tau \approx 64$.2. This (high) value of $\chi$ indicates that in the hypothetical case that the superfluid vortices were material lines in the normal flow, the latter's gradients would have deformed the vortices at a much faster rate than any deformations due to the self-induced superfluid flow. However, the superfluid vortices are not passively transported by the normal fluid, and the strong mutual friction effects will induce novel vortex tangle organizations. Our aim is to compute the vortex length dynamics and the energy spectra that correspond to these organizations, and subsequently, to compare these data with corresponding results of fully developed turbulence MM computations [11]. In this way, we hope to draw important conclusions about the structure of finite temperature superfluid turbulence. 


\section{B. Dimensional analysis of the incompressible superfluid dynamics equations}

Next, we develop a scaling framework for superfluid hydrodynamics. We start with the Reynolds number for the normal fluid $\operatorname{Re}=u d / v_{n}$ where $u$ and $d$ are characteristic normal-fluid velocities and length scales, and $v_{n}$ is the normal-fluid viscosity. Re is the ratio of inertia over viscous effects, and is equal to $\operatorname{Re}=D^{2} \dot{\epsilon} v_{n}=6.86$. Where we have used that for $T=1.3 \mathrm{~K}$ in our calculations, the normal-fluid viscosity is $v_{n}=2.33 \times 10^{-3} \mathrm{~cm}^{2} \mathrm{~s}^{-1}$. Since, in a turbulent flow, $\operatorname{Re}=O(1)$ at the small scales, we conclude that our setup is characteristic of vortex-small turbulent scale interactions. Taking into account that most turbulence strain takes place at small scales, this is a physically well motivated parameter choice. Moreover, as shown in the direct MM calculation of [11], the small scales are the key for understanding interactions between normal-fluid, high enstrophy, small-scale vortices and superfluid vortices, with the latter damping the former by absorbing their energy to increase their length $[11,25]$, while inducing instabilities that destroy the normal-fluid vortices [21]. Remarkably, as shown in [21,26], these instabilities appear in straight normal-fluid vortices, and vortex rings are not similarly affected. In actual turbulent flows, small-scale vortices are approximately straight, since they correspond to high enstrophy events, representing the end stages in the evolution of curved, larger-scale vortices which, through self-stretching, generate turbulence direct cascade events, and become straight in the process [28,33]. These remnants of the cascade process develop then instabilities due to mutual-friction interactions with the superfluid vortices [21].

Other useful nondimensional numbers are the densities ratio $\pi=\rho_{n} / \rho_{s}=0.047$, and the ratio of normal-fluid over superfluid inertia: $I=\pi u_{n} D / \kappa=\rho_{n} D^{2} \dot{\epsilon} / \rho_{s} \kappa=0.755$, where we used the characteristic normal-fluid velocity $u_{n}=D \dot{\epsilon}=0.4 \mathrm{~cm} \mathrm{~s}^{-1}$, and the ratio of densities $\rho_{n} / \rho_{s}=0.047$. Hence, at small turbulent normal-fluid scales, the inertia of the two fluids is comparable, and this is consistent with the damping action reported in [11]. We also need to compare the inertia in the two fluids with mutual friction effects. Since mutual friction depends on the difference $\mathbf{v}_{v n}$ between vortex and normal-fluid velocities [12], and the latter changes as the system evolves, one can employ the Biot-Savart velocity to characterize the vortex velocity in the initial conditions only. Accordingly, $u_{\ell}=2 \kappa / D=0.05 \mathrm{~cm} \mathrm{~s}^{-1}$. Notably, although the viscous drag related mutual friction force is directed along the $\mathbf{v}_{v n}$ velocity difference, the vacuum lift mutual friction force involves the $\sin \theta$ of the typical angle $\theta$ between $\mathbf{v}_{v n}$ and the unit tangent along the vortices. Since in the initial conditions the vortices have a random orientation with respect to $\mathbf{v}_{v n}$, a reasonable estimate is $\sin \theta \approx 0.637$, the average value of $\sin \theta$ when $\theta \in[0, \pi]$. Finally, we assume that the mutual friction length scales (delta-function smoothing scale and length scale along the superfluid vortices) are of the same order as normal-fluid length scales characterizing viscous stress gradients. These issues have been extensively discussed in [11]. Then the ratio of viscous stress over lift force is $F_{\ell}=v_{n} u_{n} / \sin \theta \kappa\left(u_{n}-u_{\ell}\right)=4.2$, and the ratio of viscous stress over drag force is $F_{d}=$ $v_{n} u_{n} / v_{c}\left(u_{n}-u_{\ell}\right)=0.5$, where $v_{c}=2.223 v_{n}$ is the drag-force coupling viscosity [12]. Evidently, viscous and mutual friction effects are of the same order, exactly as depicted in direct superfluid turbulence calculations [11,25]. These five numbers, Re, $I, F_{\ell}, F_{d}$, and $\pi$, constitute a complete scaling of superfluid hydrodynamics, since all other relations between forces are their combinations. It is important to note that, in agreement with the character of the MM, the comparisons are between normal-fluid quantities with discrete (rather than average) topological defect quantities. For example, the ratio of superfluid inertia over the lift force is $\operatorname{Re} F_{\ell} I^{-1} \pi=1.79$, and the ratio of superfluid inertia over the drag force is $\operatorname{Re} F_{d} I^{-1} \pi=0.213$. In agreement with our results, these numbers predict a significant effect of mutual friction forces on the superfluid vortex tangle, and the generation of new superfluid vorticity. Notably, the quantum of circulation $\kappa$ scales the "mesoscopic" superfluid inertia of a single topological defect. In general, the macroscopic superfluid inertia would be characterized by the large-scale superfluid velocity $u_{s}$ and the size of the flow domain $d$. The dimensionless number $S=u_{s} d / \kappa$ can be interpreted as a quantum vortex tangle structure indicating quantity, since, as suggested in [34], the structure number $S$ can be plausibly associated with tangle polarization. In contrast with $\kappa$, however, the "macroscopic" superfluid 
circulation $u_{s} d$ depends on the particular flow aspects, and it is the output of a MM calculation, rather than a quantity that could be used to scale a problem without any prior knowledge of the system. The actual $S$ values of technological or natural superfluid flows depend on the microscopic efficiency of the mutual fiction force to extract energy from the normal fluid and on the particular characteristics of the flow structure within the turbulent inertial range of the normal fluid, among others. In analogy with magnetohydrodynamics, one can define the "superfluid vortex dynamo" problem. This problem, however, is much more complicated than its magnetic analog since the dynamics of vortex lines are more complicated than those of magnetic lines. The examination of this problem is a key task for MM calculations and experimental investigations alike. Contrasting the five numbers needed for scaling superfluid dynamics, with only one needed for classical fluid dynamics, indicates the complex nature of the former as compared with the latter.

\section{FORMULA FOR SUPERFLUID VORTEX ENERGY SPECTRA IN UNBOUNDED DOMAINS}

Consider first a single vortex tube occupying the space curve $\mathbf{X}(s, t)$ at time $t$, where $s$ is a parameter along the curve. From now on we suppress the $t$ dependence. Then the vorticity field $\omega$ is given by

$$
\boldsymbol{\omega}(\mathbf{x})=\Gamma \int \frac{\gamma(|\mathbf{x}-\mathbf{X}(s)| / \sigma)}{\sigma^{3}} \frac{\partial \mathbf{X}(s)}{\partial s} d s,
$$

where $\Gamma$ is the circulation of the tube and $\gamma$ is related to the vorticity distribution within the core of the vortex tube of radius $\sigma$. The spherically symmetric function $\gamma$ has the following normalization:

$$
\int \gamma(\mathbf{y}) d \mathbf{y}=4 \pi \int \gamma(y) y^{2} d y=1
$$

The Fourier transform of $\omega$ is given by

$$
\hat{\boldsymbol{\omega}}(\mathbf{k})=\Gamma \hat{\gamma}(k \sigma) \int e^{i \mathbf{k} \cdot \mathbf{X}(s)} \frac{\partial \mathbf{X}(s)}{\partial s} d s,
$$

where we use $1 / 2 \pi$ and $e^{-i \mathbf{k} \cdot \mathbf{x}}$ factors when taking the inverse transform, and we define $k=2 \pi / \ell$, with $\ell$ a length scale. The energy spectrum is given by

$$
E(k)=\frac{1}{2(2 \pi)^{3}} \int|\hat{\boldsymbol{\omega}}|^{2} d \Omega_{k}=\frac{\Gamma^{2} \hat{\gamma}(k \sigma)^{2}}{2(2 \pi)^{3}} \iiint e^{i \mathbf{k} \cdot\left[\mathbf{X}(s)-\mathbf{X}\left(s^{\prime}\right)\right]} \frac{\partial \mathbf{X}(s)}{\partial s} \cdot \frac{\partial \mathbf{X}\left(s^{\prime}\right)}{\partial s^{\prime}} d s d s^{\prime} d \Omega_{k},
$$

where $d \Omega_{k}$ is the dimensionless, differential solid angle, and obeys the normalization

$$
\int E(k) d k=\frac{1}{2} \int \mathbf{u}(\mathbf{x}) \cdot \mathbf{u}(\mathbf{x}) d \mathbf{x}
$$

Integration over $\Omega_{k}$ gives

$$
E(k)=\frac{\Gamma^{2} \hat{\gamma}(k \sigma)^{2}}{(2 \pi)^{2}} \iint \frac{\sin \left(k\left|\mathbf{X}(s)-\mathbf{X}\left(s^{\prime}\right)\right|\right)}{k\left|\mathbf{X}(s)-\mathbf{X}\left(s^{\prime}\right)\right|} \frac{\partial \mathbf{X}(s)}{\partial s} \cdot \frac{\partial \mathbf{X}\left(s^{\prime}\right)}{\partial s^{\prime}} d s d s^{\prime} .
$$

For multiple tubes, we obtain the double sum

$$
E(k)=\sum_{i} \sum_{j} \frac{\Gamma_{i} \Gamma_{j} \hat{\gamma}_{i}\left(k \sigma_{i}\right) \hat{\gamma}_{j}\left(k \sigma_{j}\right)}{(2 \pi)^{2}} \iint \frac{\sin \left(k\left|\mathbf{X}_{i}(s)-\mathbf{X}_{j}\left(s^{\prime}\right)\right|\right)}{k\left|\mathbf{X}_{i}(s)-\mathbf{X}_{j}\left(s^{\prime}\right)\right|} \frac{\partial \mathbf{X}_{i}(s)}{\partial s} \cdot \frac{\partial \mathbf{X}_{j}\left(s^{\prime}\right)}{\partial s^{\prime}} d s d s^{\prime} .
$$

We test this formula and its algorithmic implementation by reproducing the spectrum of a single superfluid vortex ring as in $[35,36]$. Notably, the method assumes that a regularization of the singular superfluid vorticity is applied. We have employed here the algebraic smoothing kernel of [37]

$$
\gamma(y)=\frac{3}{4 \pi} \frac{1}{\left(y^{2}+1\right)^{5 / 2}}
$$




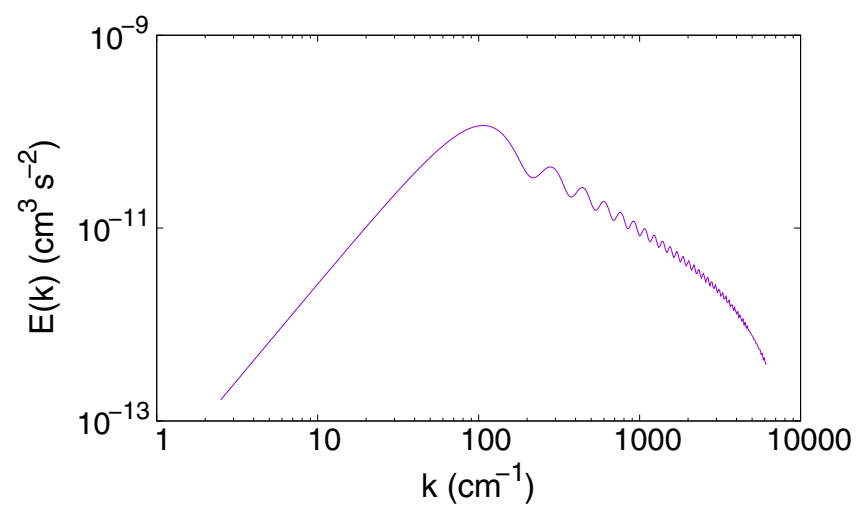

FIG. 1. Energy spectrum of a single superfluid vortex ring in helium-4. The radius of the ring is $R=$ $0.03 \mathrm{~cm}$. There are 8192 wave numbers $(k \equiv 1 / \ell)$ between length scales $\ell_{\max }=12 \mathrm{~cm}$ and $\ell_{\min }=9.76 \times$ $10^{-5} \mathrm{~cm}$.

which transforms superfluid vortices from singular lines to finite core tubes of radius $\sigma$. It is important to note that a similar method for computation of superfluid vortex spectra was employed in [38]. Our formula differs from their formula in that we perform an analytical integration over the dimensionless solid angle $\Omega_{k}$, and we include finite core effects. The latter is required by our approach of regularization of the Biot-Savart integral via smoothing of the singular delta-function vorticity over a small, albeit finite, vortex core [39]. Our method has some advantages: (a) the analytic angular integration is exact, hence, it does not need to be performed numerically and introduce truncation errors, (b) the inclusion of finite core effects makes our spectrum formula consistent with the numerical analysis, i.e., with the method employed for evolving the vortices. In other words, the spectrum computation incorporates in a consistent way the numerical smoothing of the Biot-Savart kernel. This is not necessarily a feature of other methods that regularize vortex dynamics, but assume singular vortices when computing the spectra. In spectra computations, $\sigma=0.125 \xi$, where $\xi=7.8125 \times 10^{-4} \mathrm{~cm}$ is the discretization length along the vortices. Using this value of $\xi$, a typical vortex ring in the initial conditions is discretized into $N=160$ vortex elements. The physical meaning of $\sigma$ cutoff is to indicate the scale where the superfluid velocity field is damped. The latter is achieved without introducing any dissipation in the flow, which is a particularly attractive feature of vortex methods.

Employing our algorithms (Figs. 1 and 2), we have reproduced all spectrum features in [35]: the characteristic "ringing," the low wave-number $k^{2}$ scaling corresponding to the ring's linear impulse, and the large wave-number $k^{-1}$ scaling corresponding to the isolated vortex line spectrum. The $k^{2}$ scaling has a long history in classical vortex dynamics $[40,41]$. In the context of superfluids, the $k^{2}$ regime was observed in [42], where it was correctly attributed to the $1 / r^{3}$ far-field velocity field scaling. This connection between $k^{2}$ energy spectrum scaling and $1 / r^{3}$ velocity variation was also indicated by Saffman [41]. In general, a far-field $1 / r^{3}$ velocity variation implies nonzero ring impulse.

\section{RESULTS}

Direct MM calculations of superfluid turbulence [11,25] have provided a plethora of information regarding vortex line dynamics and spectral structure. We summarize these results here: The normalfluid energy presents three spectral scaling regimes: $k^{-5 / 3}$ at small wave numbers that correspond to the Kolmogorov spectrum of classical turbulence, and where mutual-friction effects are not of the same order as inertial effects, a $k^{-2.2}$ regime at large wave numbers that corresponds to creeping flow motions around the vortices [12,18], and an intermediate cutoff $k^{-6}$ regime which, due to the 

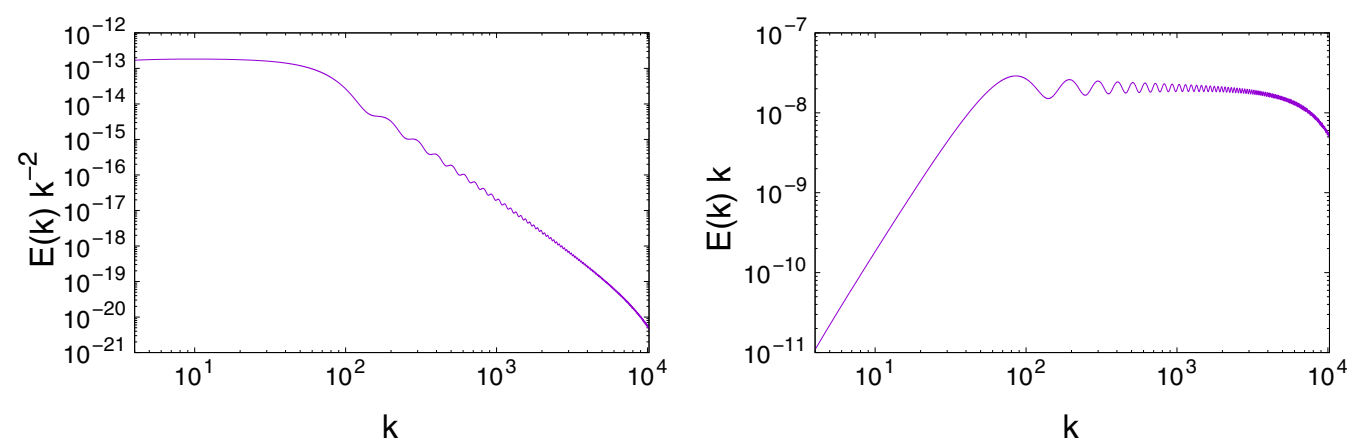

FIG. 2. Compensated energy spectrum of a single superfluid vortex ring in helium-4. There is a $k^{2}$ scaling in the small $k$ regime that corresponds to the linear impulse of the ring (left), and a $k^{-1}$ scaling at large $k$ that corresponds to the energy spectrum of an isolated line vortex (right).

presence of superfluid vortices and mutual-friction forcing, is less sharp than the exponential cutoff of classical turbulence. The corresponding scaling regimes in superfluids are $k^{-5 / 3}$ at large scales, $k^{-1}$ at small distances (isolated vortex-line spectrum), and $k^{-3}$ at intermediate scales $[11,25]$. The latter is the signature of spiral, mutual-friction induced vortex structures [25]. As shown in [25], the energy $k^{-3}$ regime corresponds to a $k^{-2}$ regime in the superfluid pressure spectra, which is the pressure spectrum of isolated vortex filaments. Due to computational complexity limitations, [11] did not have the required range of scales to conclusively determine the physical nature of the superfluid $k^{-5 / 3}$ regime. It was conjectured though, that the Kolmogorov-type spectrum in the superfluid is the result of termination of the growth of instability generated superfluid vortex structures [12,21], as "vortices emanating outwards from different instability centers (which are coherent vortical structures within the normal-fluids inertial range) collide with each other and agglomerate, in a process reminiscent of caustics formation in particulate suspensions" [11]. For completeness, it is important to note here, that not all superfluid turbulent flows are similar to those computed in $[11,25]$. In particular, there is evidence that the so-called ultraquantum turbulence of [43] does not present a Kolmogorov scaling. Overall, for reasons discussed above, previous efforts to understand the phenomenology of superfluid turbulence focused on the effects of normal-fluid vorticity. Here, we examine normal-fluid strain, and by examining length dynamics and energy spectra of superfluid vortices, we draw conclusions about the potential of these flows in explaining the phenomenology of fully developed turbulence.

As expected from our scaling analysis, all flow fields lead to vortex tangle growth (Fig. 3). Although all three curves are exponentials of the type $L \sim e^{a t}$, equibiaxial extensional flow has $a=5.7$, uniaxial extensional flow $a=3$, and shear flow $a=2.5$. Both R1 and R2 results lead to qualitatively similar conclusions. These growth rates are faster than the corresponding $L \sim t^{3}$ rate computed for vortex tangles kinematic normal-fluid turbulence calculations [25]. To understand this phenomenology better, we need to examine vortex tangle configurations and the accompanying energy spectra. Our discussion will employ intuition provided by MM superfluid vortex dynamics [12]. To exemplify this intuition, we shall consider a vortex ring moving with velocity $\dot{\mathbf{X}}_{v}$ in a normal-fluid flow with velocity $\mathbf{V}_{n}$, where $\dot{\mathbf{X}}_{v} \| \mathbf{V}_{n}$. Then the ring shrinks due to Hall-Vinen force, when it either moves against the flow, or it moves with the flow, but $\left\|\mathbf{V}_{n}\right\| \leqslant\left\|\dot{\mathbf{X}}_{v}\right\|$. The ring grows when it moves with the flow and $\left\|\mathbf{V}_{n}\right\| \geqslant\left\|\dot{\mathbf{X}}_{v}\right\|$. Moreover [12], the Iordanskii force contributes a ring velocity that opposes $\dot{\mathbf{X}}_{v}-\mathbf{V}_{n}$. Another useful intuition is the instability responsible for transferring energy from the normal-fluid to the superfluid vortices [12], which is the MM analog of the Ostermeier-Glaberson instability in ECM, and can occur when the normal-fluid velocity is parallel to the vortex contour. Notably, all calculations terminate at the same superfluid vortex tangle length. This is because computational complexity scales with the number of superfluid vortices in 

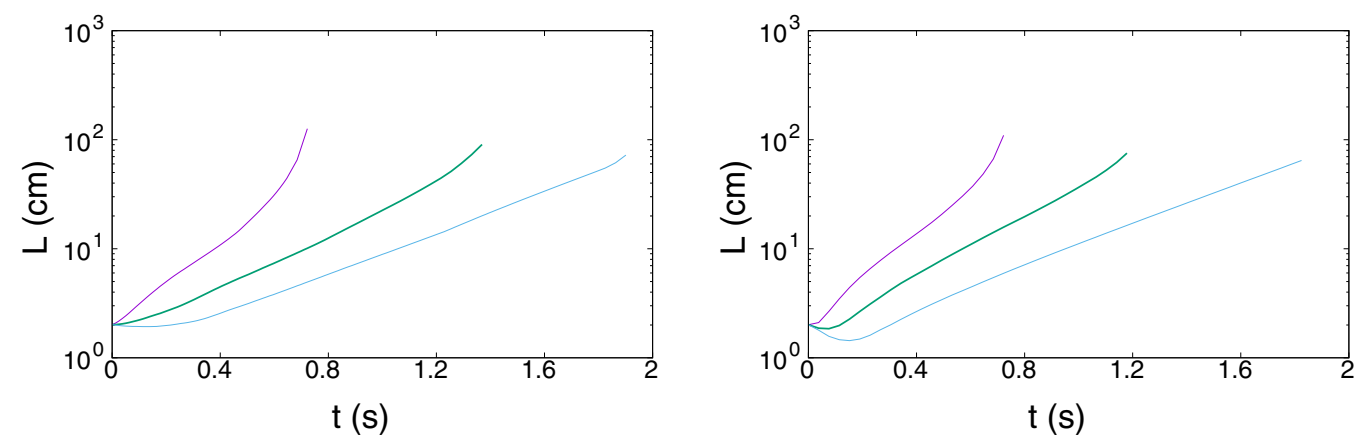

FIG. 3. Left: Superfluid vortex tangle length $L$ versus time for equibiaxial extensional flow (left curve), uniaxial extensional flow (middle curve), and simple-shear flow (right curve). Right: Same results for R2 initial conditions.

the system, and beyond this length it is not practically feasible to continue the computation. As we show below, the results are useful because the spectra achieve a steady state before the computations become too complex. Evidently, due to their different capacities for superfluid vorticity generation, the same length is produced at different times for each of the applied strain fields.

Although vortex length is an important physics indicator, we can gain further insight into normal-fluid strain effects, by computing the vortical impulse $\mathbf{I}$ and angular vortical impulse $\mathbf{L}$ of the superfluid vortex tangle. These quantities are defined as

$$
\mathbf{I}=\frac{\kappa}{2} \int \mathbf{X}_{v} \times \mathbf{X}_{v}^{\prime} d \xi, \quad \mathbf{L}=\frac{\kappa}{3} \int \mathbf{X}_{v} \times\left(\mathbf{X}_{v} \times \mathbf{X}_{v}^{\prime}\right) d \xi
$$

where $\xi$ is the length parametrization along a vortex contour, and the integrals are meant to run over the whole superfluid vortex tangle. $\mathbf{I}$ and $\mathbf{L}$ are (correspondingly) the global (or total) momentum and angular momentum of a fluid that are associated with its vorticity [44]. In our case, this is a trivial correspondence, since all superfluid flow is due to the vortex tangle. Similar to vortex length, equibiaxial strain is the most effective in imparting momentum and angular momentum to the fluid (Fig. 4, top). Interestingly, shear strain is slightly more effective than uniaxial extensional strain in augmenting I and L. Figure 4 (bottom) shows the same results for R2 initial conditions. Although the angular vortical impulse results are similar, there is an important difference in the vortical impulse data. The uniaxial extensional flow vortical impulse is much closer to the equibiaxial extensional flow curve, instead of being similar to the simple shear curve as in the R1 case. This indicates an interesting effect of vortex configurations in uniaxial extensional flow on the rate with which momentum is transferred from the normal fluid to the superfluid.

\section{A. Uniaxial extension}

The initial vortex tangle [Fig. 5(a)] is a blob of vorticity around the origin of the coordinate system. Notably, our scaling analysis has shown that Hall-Vinen (drag force) effects are larger than Iordanskii (lift force) effects by a factor 5.34. Under the influence of the lift force, the loops move towards the extensional axis (horizontal), where they become stretched. Although this would be the complete phenomenology of polymers in such a flow [27,45], resulting in a vortex filament, in superfluids, we have stronger, viscous drag effects. The latter cause the loops to grow along the extensional axis as they approach it, an effect combined with a simultaneous growth normal to the horizontal [Figs. 5(b)-5(e)], due to the outward flow field. Loop growth due to the extensional velocity is much more pronounced than that due to compressive velocity components [Fig. 5(f)], since, according to the kinematics of this flow, the latter are small close to the horizontal, while the 


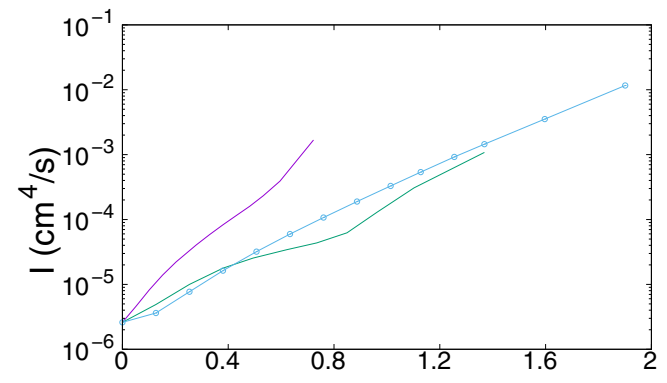

$\mathrm{t}(\mathrm{s})$

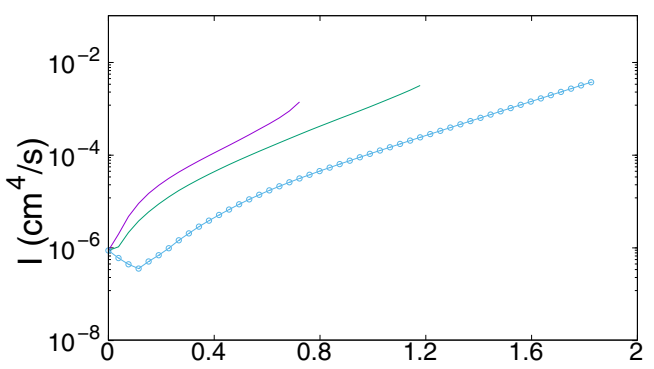

$\mathrm{t}(\mathrm{s})$

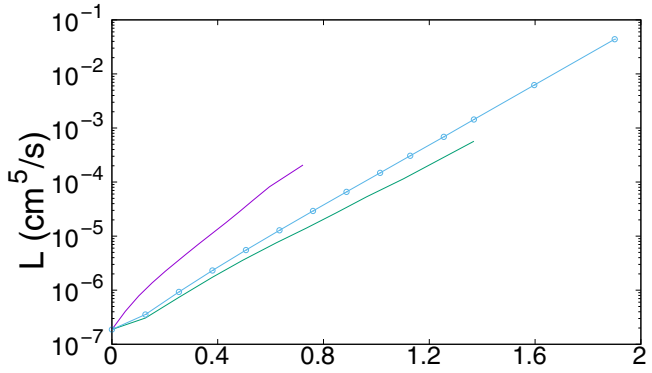

$t(s)$

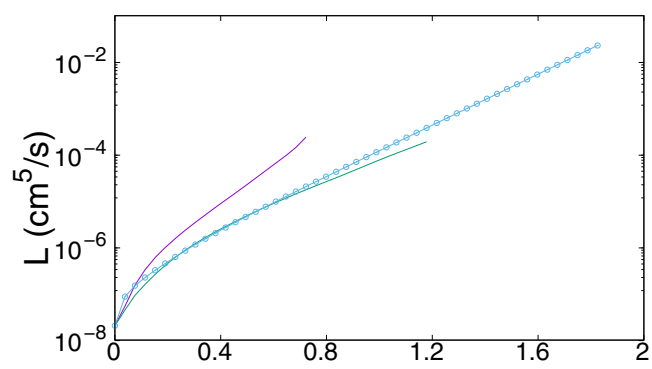

$t(s)$

FIG. 4. Top: Vortical impulse I (left) and angular vortical impulse $\mathbf{L}$ (right) magnitudes versus time. The equibiaxial flow results correspond to the leftmost curves, while the shear-flow curves are marked by circles. Bottom: Same results for R2 initial conditions.

former increases with distance from the origin. The net result is two conical surfaces connected by a narrow bridge.

We have checked that the shape of energy spectra reached a steady state before the end of the calculation (Fig. 6). In comparison with the single ring spectra, a new scaling regime of

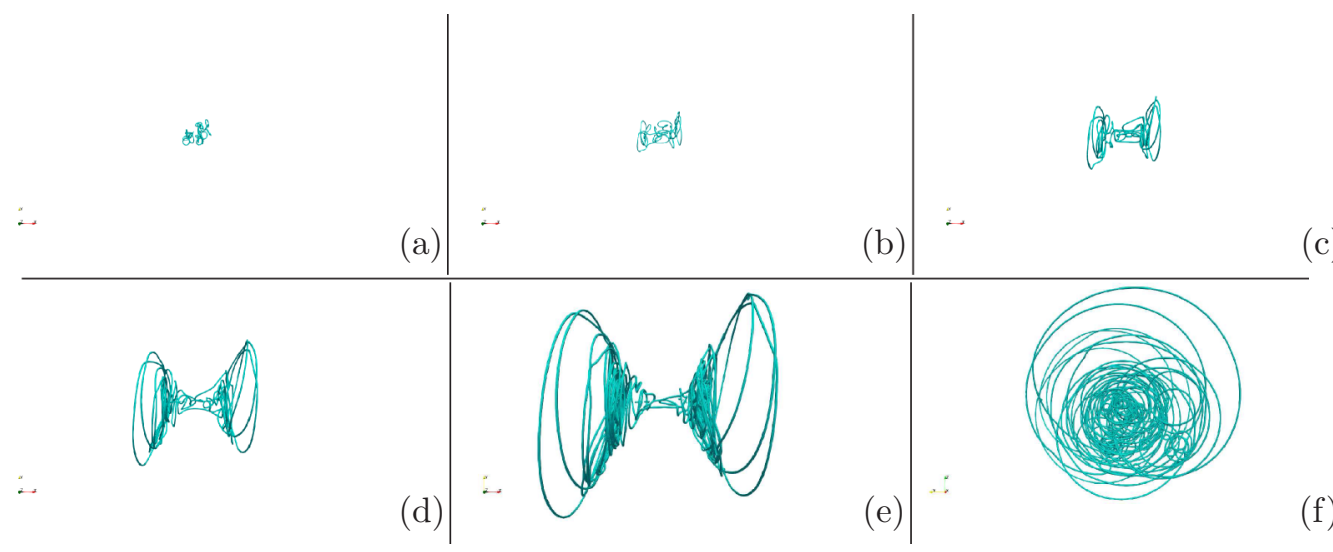

FIG. 5. Uniaxial extensional flow. (a)-(e) Evolution of superfluid vortex tangle at times $t=$ $[0,0.31,0.63,0.95,1.29] \mathrm{s}$. The horizontal is coinciding with the extensional axis. All figures are drawn with the same scale. In (f), time is the same as in (e), but the view is along the extensional axis, so that key structural details are better indicated. These vortex configurations are also reproduced in the R2 results. 

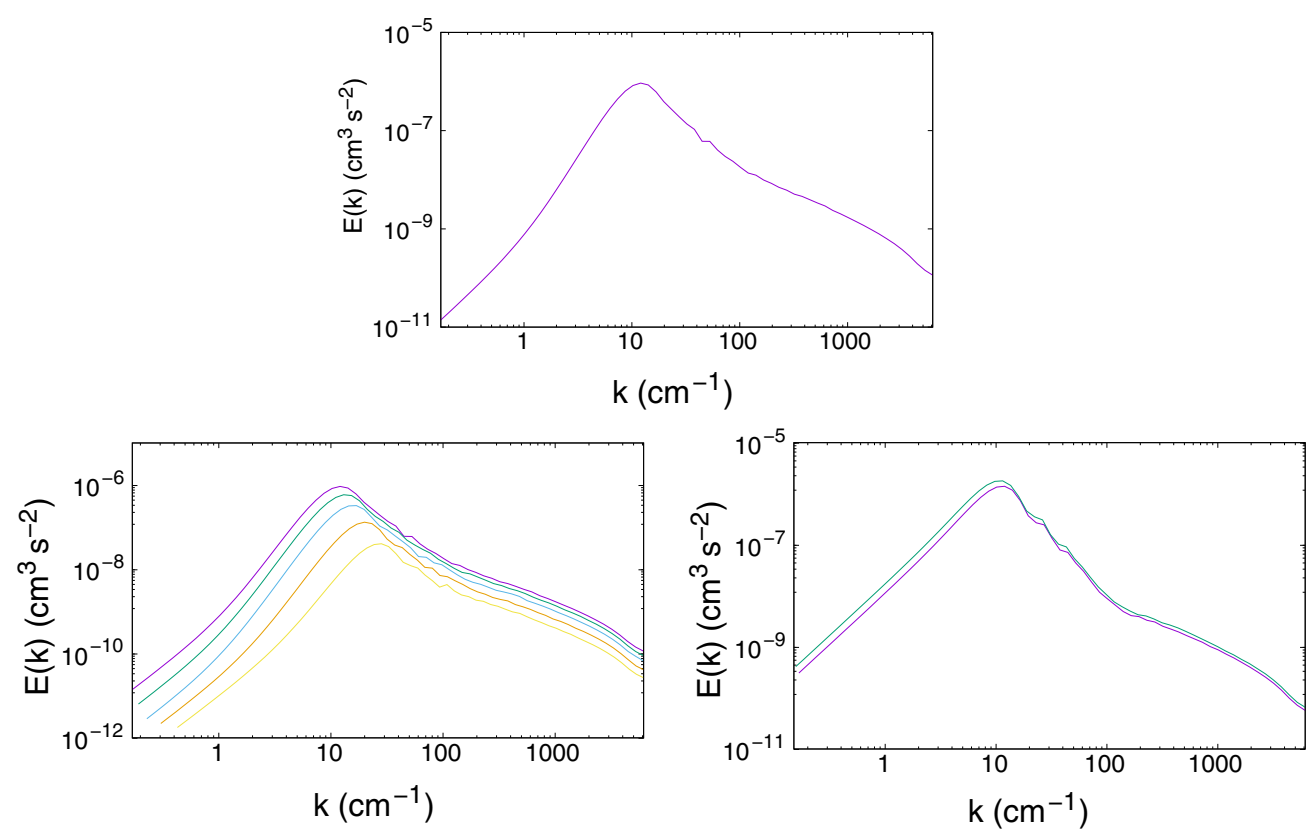

FIG. 6. Top: Energy spectrum of superfluid vortex tangles in uniaxial extension at $t=1.0 \mathrm{~s}$, after the initial transients has ceased, and the shape has reached a steady state. There are four scaling regimes: $k^{2}$ at smallest $k, k^{10 / 3}$ at larger but still small $k(k \in[1.0,5.5]), k^{-7 / 3}$ at intermediate $k(k \in[15,75])$, and $k^{-1}$ at high $k(k \in[147,1900])$. The final cutoff is due to the numerical smoothing of the Biot-Savart kernel. The numerical estimate of the end of the $k^{2}$ regime is $k_{t}=1.3$. Bottom (left): Demonstration of spectrum convergence. Spectra at five different times are shown. From the lower to the upper curves, the times are $t=[0.47,0.66,0.82,0.92,1.00] \mathrm{s}$. The last three spectrum curves allow identical scaling conclusions. Bottom (right): R2 results for times $t=[1.06,1.10] \mathrm{s}$. The scaling of the wave-number regime before the spectrum peak has switched from $k^{10 / 3}$ (in the R1 data) to $k^{7 / 3}$.

$E(k) \sim k^{-7 / 3}$ appears between the high wave-number $k^{-1}$ range, and the peak of the spectrum. Since $k^{-2}$ is the spectrum of vortex sheets, this scaling appears to correspond to the intermediate scale vortex sheets formed in the system. The small deviation from the $k^{-2}$ scaling could be related to sheet curvature effects. On the other hand, the low wave-number scaling of $k^{10 / 3}$ is much steeper than the corresponding $k^{2}$ single-ring scaling, and it is the spectral signature of the large-scale geometrical shape of the vortex tangle. It is intriguing that the surface outlined by the superfluid vortices appears to be a catenoid, which is an example of a minimal surface, i.e., a surface whose mean curvature is everywhere zero, and its area is smaller than the area of all possible surfaces bounded by the same two circles, each on different ends of the structure [46]. Such surfaces are easily realizable in soap-film experiments [46], and it is remarkable that they appear in such a different physics context here. The R2 data (Fig. 6) present the same spectral structure and scalings, with an important difference. The scaling exponent in the wave-number regime preceding the peak of the spectrum is $7 / 3$, i.e., much smaller than the $10 / 3$ exponent in the R 1 case. This could very well be the spectral analog of the difference between R1 and R2 data in the vortical impulse results. It is an interesting connection that can hint at the experimental control of superfluid vortex dynamics processes. Notably, the scaling at the smallest wave numbers is $k^{2}$. This is easy to understand by employing the Maclaurin series expansion of the sine function in our $E(k)$ formula. Then, we can 


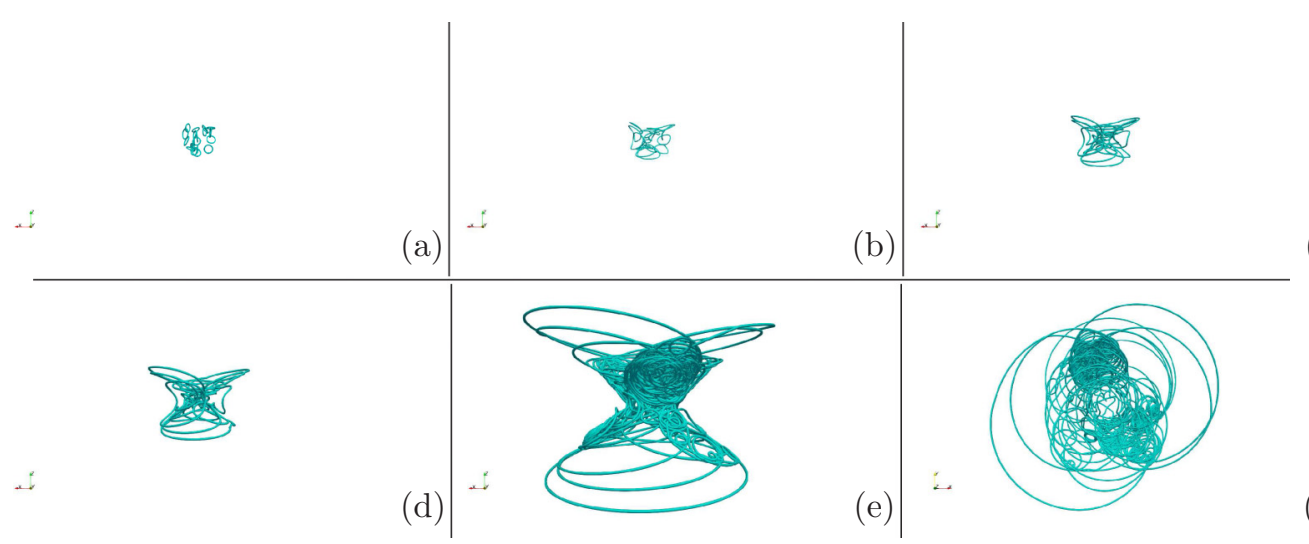

FIG. 7. Equibiaxial strain flow. (a)-(e) Evolution of superfluid vortex tangle at times $t=$ $[0,0.12,0.25,0.50,0.72] \mathrm{s}$. The vertical is coinciding with the compressive axis. All figures are drawn with the same scale. In (f), time is the same as in (e), but the view is along the compressive axis, so that key structural details are better indicated. These vortex configurations are also reproduced in the $\mathrm{R} 2$ results.

compute the sum of the coefficients of the $k^{2}$ and $k^{4}$ terms. These are

$$
\begin{gathered}
C_{k^{2}}=-\sum_{k} \sum_{i} \sum_{j} \frac{\Gamma_{i} \Gamma_{j} \hat{\gamma}_{i}\left(k \sigma_{i}\right) \hat{\gamma}_{j}\left(k \sigma_{j}\right)}{(2 \pi)^{2}} \iint \frac{\left|\mathbf{X}_{i}(s)-\mathbf{X}_{j}\left(s^{\prime}\right)\right|^{2}}{6} \frac{\partial \mathbf{X}_{i}(s)}{\partial s} \cdot \frac{\partial \mathbf{X}_{j}\left(s^{\prime}\right)}{\partial s^{\prime}} d s d s^{\prime}, \\
C_{k^{4}}=\sum_{k} \sum_{i} \sum_{j} \frac{\Gamma_{i} \Gamma_{j} \hat{\gamma}_{i}\left(k \sigma_{i}\right) \hat{\gamma}_{j}\left(k \sigma_{j}\right)}{(2 \pi)^{2}} \iint \frac{\left|\mathbf{X}_{i}(s)-\mathbf{X}_{j}\left(s^{\prime}\right)\right|^{4}}{120} \frac{\partial \mathbf{X}_{i}(s)}{\partial s} \cdot \frac{\partial \mathbf{X}_{j}\left(s^{\prime}\right)}{\partial s^{\prime}} d s d s^{\prime} .
\end{gathered}
$$

Since $E=C_{k^{2}} k^{2}+C_{k^{4}} k^{4}+O\left(k^{6}\right)$ for $k \ll 1 / \ell$, where $\ell$ is the size of the large-scale flow motions, the $k^{2}$ scaling should appear below the scale $k_{t}=\sqrt{C_{k^{2}} / C_{k^{4}}}$. Indeed, in all cases, there is very good agreement between the actual transition wave numbers, and the corresponding $k_{t}$ values computed with these formulas (Figs. 6, 8, and 11).

The fact that all spectra present a low wave-number $k^{2}$ regime, allows one to conclude that the vortical impulse $I$ is nonzero. This connection emanates from the following analysis of the double integral in the expression for $C_{k^{2}}$ :

$$
\mathcal{K}=\sum_{i} \sum_{j} \iint\left|\mathbf{X}_{i}(s)-\mathbf{X}_{j}\left(s^{\prime}\right)\right|^{2} \frac{\partial \mathbf{X}_{i}(s)}{\partial s} \cdot \frac{\partial \mathbf{X}_{j}\left(s^{\prime}\right)}{\partial s^{\prime}} d s d s^{\prime} .
$$

Employing the identities $\quad\left|\mathbf{X}_{i}(s)-\mathbf{X}_{j}\left(s^{\prime}\right)\right|^{2}=\left|\mathbf{X}_{i}(s)\right|^{2}+\left|\mathbf{X}_{j}\left(s^{\prime}\right)\right|^{2}-2 \mathbf{X}_{i}(s) \cdot \mathbf{X}_{j}\left(s^{\prime}\right) \quad$ and $\oint[\partial \mathbf{X}(s) / \partial s] d s \equiv 0$, we have

$$
\mathcal{K}=-2 \sum_{i} \sum_{j} \iint\left[\mathbf{X}_{i}(s) \cdot \mathbf{X}_{j}\left(s^{\prime}\right)\right]\left[\frac{\partial \mathbf{X}_{i}(s)}{\partial s} \cdot \frac{\partial \mathbf{X}_{j}\left(s^{\prime}\right)}{\partial s^{\prime}}\right] d s d s^{\prime} .
$$

Applying the Binet-Cauchy vector identity

$$
\begin{aligned}
\left(\mathbf{X}_{i}(s) \times \frac{\partial \mathbf{X}_{i}(s)}{\partial s}\right) \cdot\left(\mathbf{X}_{j}\left(s^{\prime}\right) \times \frac{\partial \mathbf{X}_{j}\left(s^{\prime}\right)}{\partial s^{\prime}}\right)= & {\left[\mathbf{X}_{i}(s) \cdot \mathbf{X}_{j}\left(s^{\prime}\right)\right]\left[\frac{\partial \mathbf{X}_{i}(s)}{\partial s} \cdot \frac{\partial \mathbf{X}_{j}\left(s^{\prime}\right)}{\partial s^{\prime}}\right] } \\
& -\left(\frac{\partial \mathbf{X}_{i}(s)}{\partial s} \cdot \mathbf{X}_{j}\left(s^{\prime}\right)\right)\left(\mathbf{X}_{i}(s) \cdot \frac{\partial \mathbf{X}_{j}\left(s^{\prime}\right)}{\partial s^{\prime}}\right)
\end{aligned}
$$



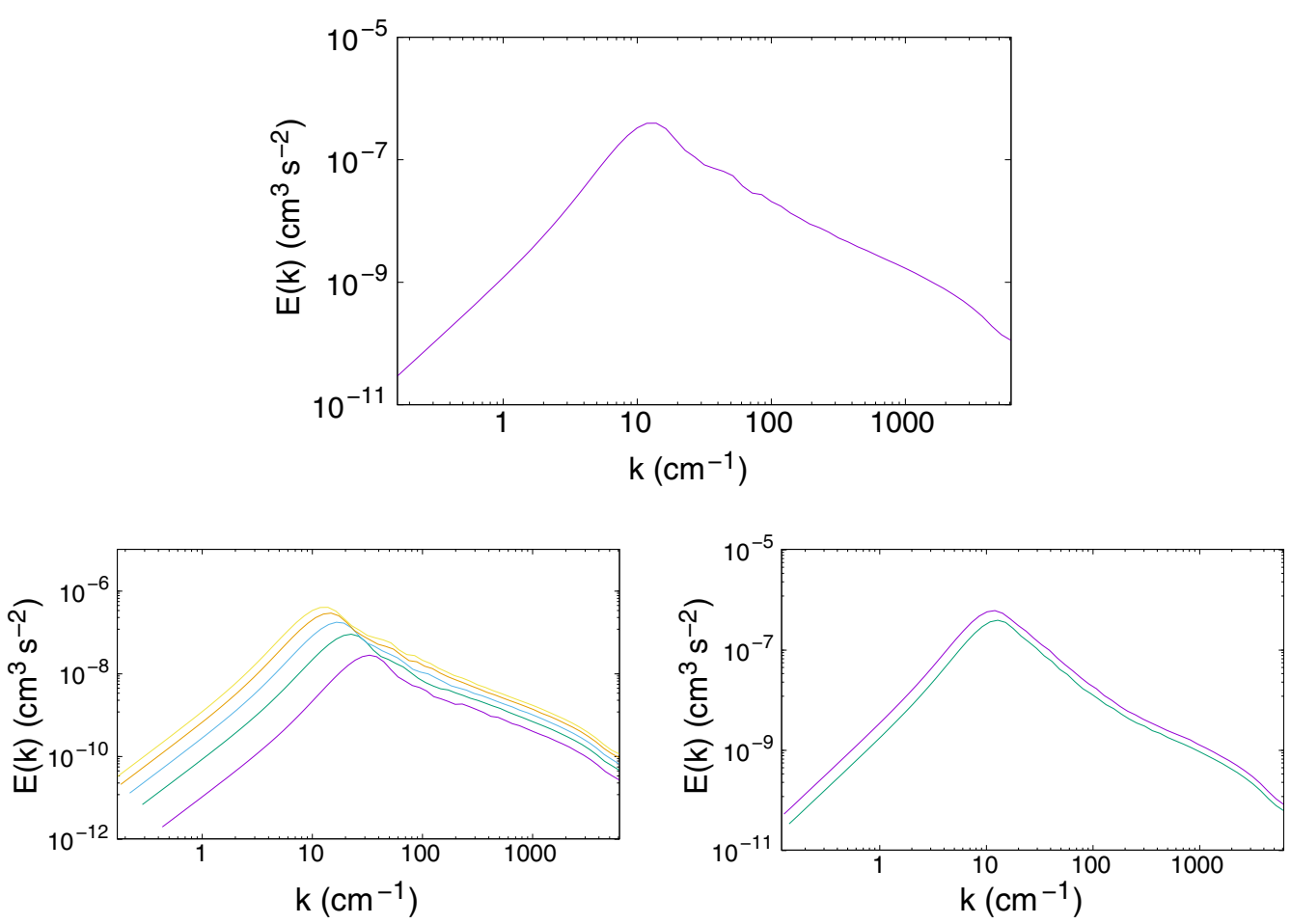

FIG. 8. Top: Energy spectrum of superfluid vortex tangles in equibiaxial extension at $t=0.54 \mathrm{~s}$, after the initial transients have ceased, and the shape has reached a steady state. There are four scaling regimes: $k^{2}$ at smallest $k, k^{10 / 4}$ at larger but still small $k(k \in[3,7.5]), k^{-9 / 5}$ at intermediate $k(k \in[15,70])$, and $k^{-1}$ at high $k(k \in[170,1500])$. The final cutoff is due to the numerical smoothing of the Biot-Savart kernel. The numerical estimate of the end of the $k^{2}$ regime is $k_{t}=3.6$. Bottom (left): Demonstration of spectrum convergence. Spectra at five different times are shown. From the lower to the upper curves, the times are $t=[0.21,0.35,0.44,0.50,0.54] \mathrm{s}$. The last three spectrum curves allow identical scaling conclusions. Bottom (right). R2 results for times $t=[0.68,0.64] \mathrm{s}$. The scalings are identical with R1 scalings. However, the $k^{-9 / 5}$ scaling at intermediate $k$ is now observed over a wider wave-number interval $[15,185]$.

and inserting into $\mathcal{K}$, the last group of terms in the Binet-Cauchy identity results in zero, and so $\mathcal{K}=-2(2 I / \kappa)^{2}$, which makes the connection between $I$ and the $k^{2}$ scaling regime evident.

\section{B. Equibiaxial extension}

The resulting vortex tangle structure resembles the corresponding one for uniaxial extensional flow [Figs. 7(a)-7(e), where the compressive direction coincides with the vertical]. The physical explanation with respect to the effect of viscous drag and lift forces is also similar. The key difference is that since there are two extensional directions, the bridge connecting the two conical structures is thicker. In a sense, the Hall-Vinen force due to the two extensional velocity components, expands loops and expels them along the vertical, "feeding" the Hall-Vinen force induced growth effect of the incoming compressive flow [Fig. 7(f)]. Without the insight provided by superfluid vortex dynamics, and by only employing experience from material lines in a flow, it would not have been possible to understand the emerging vortex structure geometry.

We have checked that the shape of energy spectra reached a steady state before the end of the calculation (Fig. 8). In comparison with the single-ring spectra, a new (short in wave-number space) scaling regime of $E(k) \sim k^{-9 / 5}$ appears between the high wave-number $k^{-1}$ range, and the peak of 


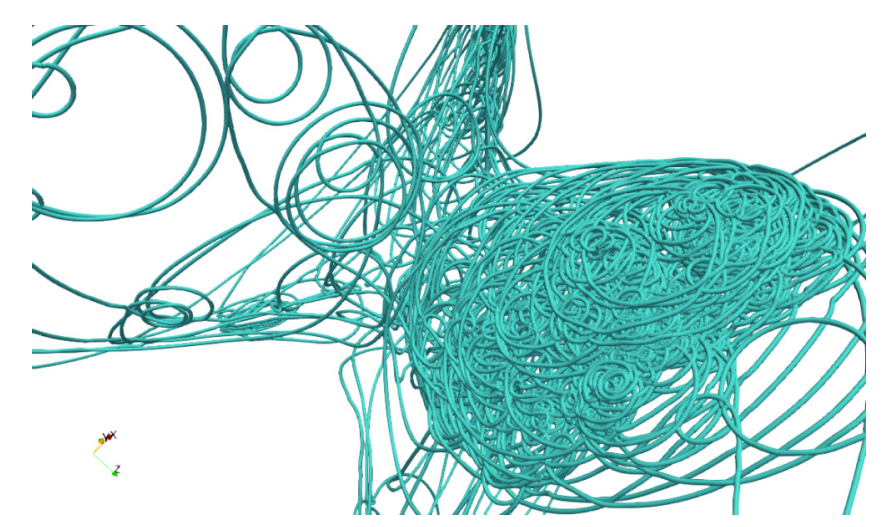

FIG. 9. Superfluid vortex sheetlike structure formation $(t=0.72 \mathrm{~s})$ in equibiaxial extensional flow. The sheets have a fractal structure, composed of a hierarchy of quasicircular loops one within the other.

the spectrum. Moreover, the low wave-number scaling of $k^{10 / 4}$ is steeper than the corresponding $k^{2}$ single-ring scaling. The 10/4 exponent could be the spectral signature of the large-scale shape of the vortex structure, which appears to be a physical analog of geometrical one-sheeted hyperboloids [47]. On the other hand, the observed smaller-scale hierarchical patterns (Fig. 9) are unique examples of fractal vortex sheets in hydrodynamics of spin-0 superfluids (where the topological defects are linear), and could be the focus of future experimental investigations. Their strikingly self-similar features might be responsible for the difference between the $E(k) \sim k^{-9 / 5}$ scaling here, and the $E(k) \sim k^{-7 / 3}$ scaling observed in the uniaxial case. As explained above, we also observe the anticipated $k^{2}$ scaling at the smallest wave numbers. The $\mathrm{R} 2$ spectral scalings are identical with those of $\mathrm{R} 1$, but the $E(k) \sim k^{-9 / 5}$ scaling is now valid over a wider wave-number range. This hints at an interdependence between the support of the $k^{-9 / 5}$ scaling and the size of the vortex structures.

\section{Simple shear}

Simple-shear strain is a two-dimensional flow, with only one nonzero velocity component along the $x$ axis, that varies linearly along the $y$ axis. There is different flow direction for positive and negative $y$ coordinates. The most important dynamical effect [Figs. 10(a)-10(d)] is loop growth normal to the flow, due to drag force action. There are two distinct areas of growth separated by a nexus corresponding to the origin of the coordinate system. Because of the linear velocity variation, greater distances from the nexus correspond to larger velocities, hence to larger growth, and larger loops. On the other hand, the lift force tends to displace the loops along the $x$ direction, and the direction of this displacement depends on the direction of flow or (equivalently) on the sign of the $y$ coordinate [Figs. 10(e) and 10(f)]. It is easy to verify [27] that simple shear can be decomposed into pure strain (along a line of slope $45^{\circ}$ ) with rate $\dot{\epsilon} / 2$, and pure rotation with rate $\dot{\epsilon} / 2$. Our scaling indicates small Iordanskii force effects, hence, there are only weak signs of vortex tilting [Fig. 10(e)], and the phenomenology is dominated by the Hall-Vinen force.

We have checked that the shape of energy spectra reached a steady state before the end of the calculation (Fig. 11). In comparison with the single-ring spectra, a new scaling regime of $E(k) \sim$ $k^{-5 / 3}$ appears between the high wave-number $k^{-1}$ range, and the peak of the spectrum. It does not appear to be a direct connection between this scaling and the large-scale $k^{-5 / 3}$ energy-spectra regime of fully developed turbulence in the superfluid component [11]. Moreover, the low wave-number scaling of $k^{12 / 5}$ is only slightly steeper than the corresponding $k^{2}$ single-ring scaling, which can be explained by the absence of any novel geometrical shape, and the typically circular rings that make up the tangle. As explained above, we also observe the anticipated $k^{2}$ scaling at the smallest wave numbers. R2 scaling features are identical with those in the R1 data. 


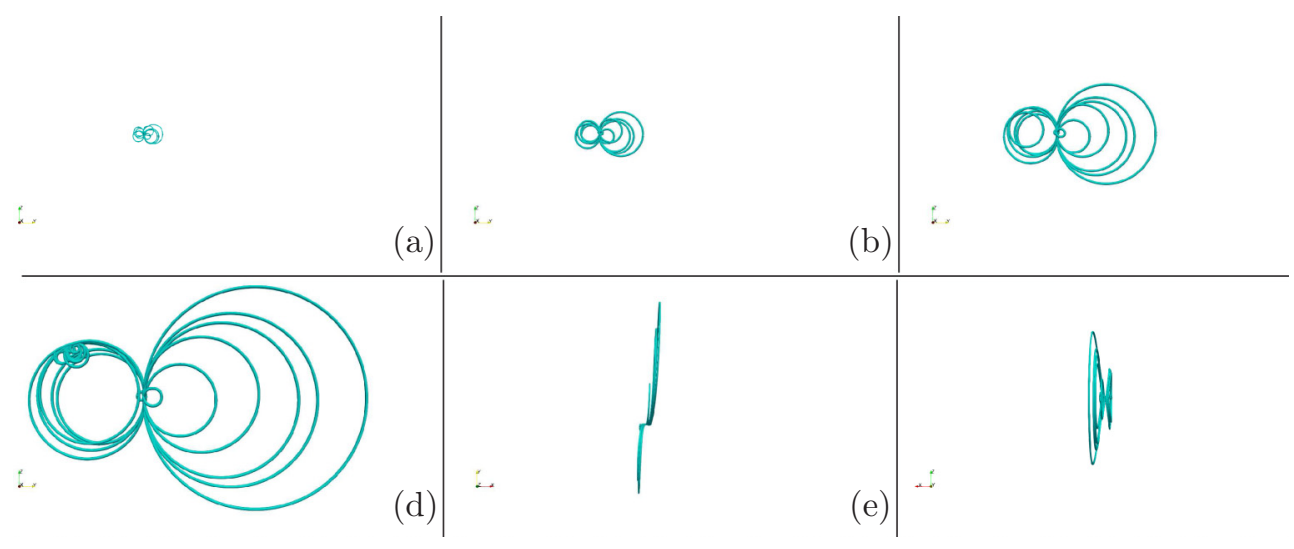

(c)

FIG. 10. Simple shear. (a)-(d) Evolution of superfluid vortex tangle at times $t=[0.76,1.14,1.52,1.90] \mathrm{s}$. The graphs show the tangle's projection on the plane normal to the direction of velocity ( $x$ direction). All figures are drawn with the same scale. In (e) and (f), time is the same as in (d). The view in (e) is along the axis which is normal to the plane of shear ( $z$ direction), and ( $f$ ) along the direction of velocity gradient ( $y$ axis). These vortex configurations are also reproduced in the $\mathrm{R} 2$ results.
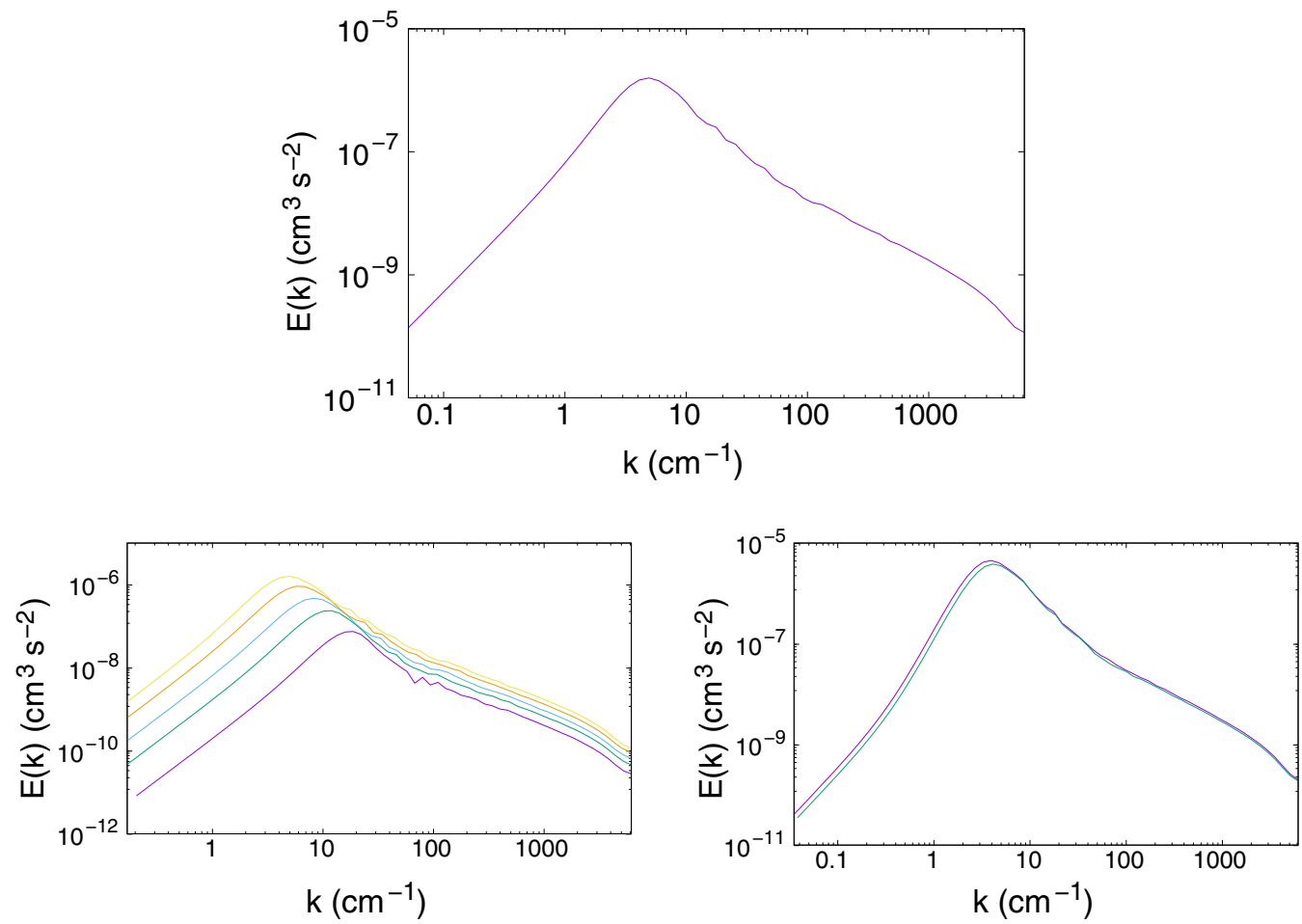

FIG. 11. Top: Energy spectrum of superfluid vortex tangles in simple shear at $t=1.41 \mathrm{~s}$, after the initial transients have ceased, and the shape has reached a steady state. There are four scaling regimes: $k^{2}$ at smallest $k, k^{12 / 5}$ at larger but still small $k(k \in[1,8]), k^{-1.6}$ at intermediate $k(k \in[7.5,67])$, and $k^{-1}$ at high $k(k \in[192,1640])$. The final cutoff is due to the numerical smoothing of the Biot-Savart kernel. The numerical estimate of the end of the $k^{2}$ regime is $k_{t}=1.8$. Bottom (left): Demonstration of spectrum convergence. Spectra at five different times are shown. From the lower to the upper curves, the times are $t=[0.21,0.35,0.44,0.50,0.54] \mathrm{s}$. The last three spectrum curves allow identical scaling conclusions. Bottom (right): $\mathrm{R} 2$ results for times $t=[1.59,1.55] \mathrm{s}$. The scalings are identical with $\mathrm{R} 1$ scalings. 


\section{CONCLUSIONS}

Direct numerical calculations with the MM indicated the spectral and vortex structure of finite temperature superfluid temperature $[11,25]$. Subsequently, there has been success in explaining this phenomenology in terms of interactions between vortex structures in the two fluids [12,21], that reconfirmed the key role of vorticity in turbulence physics [28]. However, the conceptual analogy between complex fluids and superfluids suggests that strain (and not only vortical) fields could also play an important role in superfluid turbulence generation. However, the present investigation indicates that this is not the case: simple (normal-fluid) strain fields generate superfluid vortex structures and accompanying spectra that are not observed in turbulence calculations. Indeed, none of the new energy spectra scalings match (for example) the intermediate $k^{-3}$ superfluid turbulence scaling [11], that has been shown to correspond to interactions between normal-fluid vortex tubes and superfluid loops [21]. The lack of evidence of strain effects on the phenomenology of superfluid turbulence could, perhaps, be understood by reconsidering the comment in [11]: "vortices emanating outwards from different instability centers (which are coherent vortical structures within the normalfluids inertial range) collide with each other and agglomerate, in a process reminiscent of caustics formation in particulate suspensions." Thus, new superfluid vortices are generated within vortical rather than strain normal-flow fields, and propagate away from the instability centers [11]. In the process, they gradually lose their curvature, hence, by the time they pass through turbulent flow stagnation points (similar to the ones considered here), they resemble more straight vortices rather than the rings in the initial conditions that we employed here.

Another important point is that, although vortex interactions in the two fluids explain very well the generation of turbulence in the superfluid via energy transfer from the normal fluid [12,21], there is no corresponding chaotic tangle formation in the simple strain field cases. The resulted patterns are too ordered, and do not agree with emerging structures in actual turbulent solutions. Indeed, the simple-strain fields and their accompanying vortex growth mechanisms appear to iron out any posssible Ostermeier-Glaberson type of instabilities, that are so instrumental in superfluid tubulence generation $[11,12,25]$.

On the other hand, the simple-strain fields could be employed in fundamental experimental studies of superfluid hydrodynamics [48-50]. Indeed, the difference in intuition between the behavior of polymers or colloids in a normal fluid on one hand, and that of superfluid vortices on the other, could provide an excellent experimental test of the MM and superfluid defect dynamics in general. Moreover, these flow fields could be applied to simple devices employing superfluid flow. Similarly, the fractal vortex sheets of equibiaxial extension are very interesting structures by themselves. In this respect, it will be useful to introduce, in future investigations, the feedback from the superfluid to the normal fluid.

We have also performed a comparative analysis between different initial conditions. We have shown that, overall, the results are highly reproducible. There is formation of similar vortex structures and there are similar vortex length dynamics. The spectral structure is also reproducible including most of the corresponding scaling exponents. However, we have demonstrated that, in the uniaxial extensional flow case, linear impulse results can depend on the initial conditions and this could affect the scaling exponent in the wave-number regime preceding the spectrum peak. This indicates an interesting effect of vortex configuration in uniaxial extensional flow on the rate with which momentum is transferred from the normal fluid to the superfluid, and hints at an important role that these measures might play in superfluid vortex dynamics. This feature could be employed by experimentalists to control the physics of such structures.

\section{ACKNOWLEDGMENT}

The authors are grateful to R. Hänninen for indicating to them Ref. [29]. 
[1] B. Gripaios and D. Sutherland, Quantum Field Theory of Fluids, Phys. Rev. Lett. 114, 071601 (2015).

[2] S. K. Nemirovskii, Quantum turbulence: Theoretical and numerical problems, Phys. Rep. 524, 85 (2013).

[3] Quantized Vortex Dynamics and Superfluid Turbulence, edited by C. F. Barenghi and R. J. Donnelly (Springer, Berlin, 2010).

[4] R. J. Donnelly, Quantized Vortices in Helium II (Cambridge University Press, Cambridge, 2008).

[5] W. F. Vinen, Mutual friction in a heat current in liquid helium II. II. Experiments on transient effects, Proc. R. Soc. London, Ser. A 240, 128 (1957).

[6] G. E. Volovik, Three nondissipative forces on a moving vortex line in superfluids and superconductors, Pis'ma Zh, Eskp. Teor. Fiz. 62, 58 (1995) [JETP Lett. 62, 65 (1995)].

[7] K. W. Schwarz, Three-dimensional vortex dynamics in superfluid ${ }^{4} \mathrm{He}$ : Line-line and line-boundary interactions, Phys. Rev. B 31, 5782 (1985).

[8] A. J. Legget, Quantum Liquids: Bose Condensation and Cooper Pairing in Condensed-Matter Systems (Oxford University Press, Oxford, 2006).

[9] E. Zaremba, A. Griffin, and T. Nikuni, Bose-Condensed Gases at Finite Temperatures (Cambridge University Press, Cambridge, 2009).

[10] B. Jackson, N. P. Proukakis, C. F. Barenghi, and E. Zaremba, Finite-temperature vortex dynamics in Bose-Einstein condensates, Phys. Rev. A 79, 053615 (2009).

[11] D. Kivotides, Energy spectra of finite temperature superfluid helium-4 turbulence, Phys. Fluids 26, 105105 (2014).

[12] D. Kivotides, Superfluid hydrodynamics with discrete topological defects, Phys. Rev. Fluids 3, 104701 (2018).

[13] V. A. Andryushchenko and L. P. Kondaurova, Energy dissipation after single vortex reconnection in He II at different temperatures, Low Temp. Phys. 45, 901 (2019).

[14] D. Jou, M. S. Mongiovi, and M. Sciacca, Hydrodynamic equations of anisotropic, polarized and inhomogeneous superfluid vortex tangles, Physica D 240, 249 (2011).

[15] L. Biferale, D. Khomenko, V. Lvov, A. Pomyalov, I. Procaccia, and G. Sahoo, Turbulent statistics and intermittency enhancement in coflowing superfluid He-4, Phys. Rev. Fluids 3, 024605 (2018).

[16] S. Yui, H. Kobayashi, M. Tsubota, and W. Guo, Fully Coupled Two-Fluid Dynamics in Superfluid ${ }^{4}$ He: Anomalous Anisotropic Velocity Fluctuations in Counterflow, Phys. Rev. Lett. 124, 155301 (2020).

[17] A. Sourie and N. Chamel, Generalization of the Kutta Joukowski theorem for the hydrodynamic forces acting on a quantized vortex, Int. J. Mod. Phys. B 34, 2050099 (2020).

[18] D. Kivotides, C. F. Barenghi, and D. C. Samuels, Triple vortex ring structure in superfluid helium II, Science 290, 777 (2000).

[19] O. M. Dix and R. J. Zieve, Vortex simulations on a 3-sphere, Phys. Rev. Research 1, 033201 (2019).

[20] G. E. Volovik, The Universe in a Helium Droplet (Oxford University Press, Oxford, 2003).

[21] D. Kivotides, Mutual-friction induced instability of normal-fluid vortex tubes in superfluid helium-4, Phys. Lett. A 382, 1481 (2018).

[22] L. Galantucci, A. W. Baggaley, N. G. Parker, and C. F. Barenghi, Crossover from interaction to driven regimes in quantum vortex reconnections, Proc. Natl. Acad. Sci. USA 116, 12204 (2019).

[23] R. Hänninen, N. Hietale, and H. Salman, Helicity within the vortex filament model, Sci. Rep. 6, 37571 (2016).

[24] The Philosophy of Vacuum, edited by S. Saunders and H. R. Brown (Clarendon Press, Oxford, 2002).

[25] D. Kivotides, Spreading of superfluid vorticity clouds in normal-fluid turbulence, J. Fluid Mech. 668, 58 (2011).

[26] D. Kivotides, Interactions between normal-fluid and superfluid vortex rings in helium-4, Europhys. Lett. 112, 36005 (2015).

[27] R. I. Tanner, Engineering Rheology (Oxford University Press, Oxford, 2000).

[28] P. Davidson, Turbulence (Oxford University Press, Oxford, 2004).

[29] E. B. Sonin, Dynamics of Quantised Vortices in Superfluids (Cambridge University Press, Cambridge, 2016).

[30] L. Thompson and P. C. E. Stamp, Quantum Dynamics of a Bose Superfluid Vortex, Phys. Rev. Lett. 108, 184501 (2012). 
[31] D. Kivotides, Gravitomagnetic effects on turbulence physics, arXiv:2005.10871 [Int. J. Mod. Phys. C (to be published)].

[32] R. Healey, Gauging What's Real: The Conceptual Foundations of Contemporary Gauge Theories (Oxford University Press, Oxford, 2009).

[33] D. Kivotides, Coherent Structure Formation in Turbulent Thermal Superfluids, Phys. Rev. Lett. 96, 175301 (2006).

[34] G. E. Volovik, Classical and quantum regimes of superfluid turbulence, JETP Lett. 78, 533 (2003).

[35] A. Leonard, Computing three dimensional incompressible flows with vortex elements, Annu. Rev. Fluid Mech. 17, 523 (1985).

[36] A. Leonard, Numerical simulation of interacting, three-dimensional vortex filaments, in Proceedings of the Fourth International Conference on Numerical Methods in Fluid Dynamics, edited by R. D. Richtmyer, Lecture Notes in Physics, Vol. 35 (Springer, Berlin, Heidelberg, 1975).

[37] G. S. Winckelmans and A. Leonard, Contributions to vortex particle methods for the computation of three-dimensional incompressible unsteady flows, J. Comput. Phys. 109, 247 (1993).

[38] T. Araki, M. Tsubota, and S. K. Nemirovskii, Energy Spectrum of Superfluid Turbulence with No NormalFluid Component, Phys. Rev. Lett. 89, 145301 (2002).

[39] D. Kivotides and S. L. Wilkin, Elementary vortex processes in thermal superfluid turbulence, J. Low Temp. Phys. 156, 163 (2009).

[40] O. M. Phillips, The final period of decay of non-homogeneous turbulence, Math. Proc. Cambridge Philos. Soc. 52, 135 (1956).

[41] P. G. Saffman, The large-scale structure of homogeneous turbulence, J. Fluid Mech. 27, 581 (1967).

[42] S. K. Nemirovskii, Energy spectrum of the quantum vortices configurations, Low Temp. Phys. 41, 478 (2015).

[43] P. M. Walmsley and A. I. Golov, Quasiclassical and Ultraquantum Decay of Superfluid Turbulence, Phys. Rev. Lett. 100, 245301 (2008).

[44] J.-Z. Wu, H.-Y. Ma, and M.-D. Zhou, Vorticity and Vortex Dynamics (Springer, Berlin, 2006).

[45] D. Kivotides, V. V. Mitkin, and T. G. Theofanous, Mesoscopic dynamics of polymer chains in high strain rate extensional flows, J. Non-Newtonian Fluid Mech. 161, 69 (2009).

[46] K. Tapp, Differential Geometry of Curves and Surfaces (Springer International Publishing, Switzerland, 2016).

[47] B. Odehnal, H. Stachel, and G. Glaeser, The Universe of Quadrics (Springer, Berlin, 2020).

[48] H. Sanavandi, S. Bao, Y. Zhang, R. Keijzer, W. Guo, and L. N. Cattafesta, A cryogenic-helium pipe flow facility with unique double-line molecular tagging velocimetry capability, Rev. Sci. Instrum. 91, 053901 (2020).

[49] V. B. Eltsov, R. Hänninen, and M. Krusius, Quantum turbulence in superfluids with wall-clamped normal component, Proc. Natl. Acad. Sci. USA 111, 4711 (2014).

[50] G. P. Bewley, D. P. Lathrop, and K. R. Sreenivasan, Superfluid helium: Visualization of quantized vortices, Nature (London) 441, 588 (2006). 